\title{
A common fixed point theorem for multivalued monotone mappings in ordered partial metric spaces
}

Shaban Sedghi, Nabiollah Shobkolaei, and Duran Turkoglu 


\title{
A COMMON FIXED POINT THEOREM FOR MULTIVALUED MONOTONE MAPPINGS IN ORDERED PARTIAL METRIC SPACES
}

\author{
SHABAN SEDGHI, NABIOLLAH SHOBKOLAEI, AND DURAN TURKOGLU
}

Received September 15, 2011

\begin{abstract}
In this paper, we use the order relation on partial metric spaces defined by [1] to prove some new fixed point theorems for multivalued monotone mappings in ordered partial metric spaces.
\end{abstract}

2000 Mathematics Subject Classification: 54H25; 47H10

Keywords: fixed point, partial metric

\section{INTRODUCTION}

In the last years, the extension of the theory of fixed point to generalized structures as cone metrics, partial metric spaces and quasi-metric spaces has received a lot of attention. One of the most interesting is partial metric space. Partial metric space is a generalized metric space in which each object does not necessarily have to have a zero distance from itself [8]. A motivation behind introducing the concept of a partial metric was to obtain appropriate mathematical models in the theory of computation and, in particular, give a modified version of the Banach contraction principle, more suitable in this context [8]. Subsequently, Valero [11], Oltra and Valero [9] and Altun et al [4] gave some generalizations of the result of Matthews. Romaguera [10] proved the Caristi type fixed point theorem on this space. Lately, I. Altun and M. Imdad [1] have introduced a partial ordering on uniform spaces utilizing $E$ - distance function and have used the same to prove a fixed point theorem for single-valued non-decreasing mappings on ordered uniform spaces. In this paper, we use the partial ordering on partial metric spaces which is defined by [1], so we prove some fixed point theorems of multivalued monotone mappings which are given for ordered metric spaces in [12] on ordered uniform spaces.

First, we recall some definitions and results needed in the sequel. The reader interested in fixed point theory in partial metric spaces is referred to the work of $[2,6,8-11]$ and references therein. 
In what follows $\mathbb{N}$ will denote the set of all natural numbers and $\mathbb{R}^{+}$the set of all positive real numbers.

A partial metric on a nonempty set $X$ is a mapping $p: X \times X \rightarrow \mathbb{R}^{+}$such that for all $x, y, z \in X$ :

$\left(\mathrm{p}_{1}\right) x=y$ if and only if $p(x, x)=p(x, y)=p(y, y)$,

$\left(\mathrm{p}_{2}\right) \quad p(x, x) \leq p(x, y)$,

( $\left.\mathrm{p}_{3}\right) \quad p(x, y)=p(y, x)$,

( $\left.\mathrm{p}_{4}\right) \quad p(x, y) \leq p(x, z)+p(z, y)-p(z, z)$.

A partial metric space is a pair $(X, p)$ such that $X$ is a nonempty set and $p$ is a partial metric on $X$. It is clear that, if $p(x, y)=0$, then from $\left(\mathrm{p}_{1}\right)$ and $\left(\mathrm{p}_{2}\right) x=y$. But if $x=y, p(x, y)$ may not be 0 . A basic example of a partial metric space is the pair $\left(\mathbb{R}^{+}, p\right)$, where $p(x, y)=\max \{x, y\}$ for all $x, y \in \mathbb{R}^{+}$. Other examples of the partial metric spaces which are interesting from a computational point of view may be found in [7] and [8].

Let $(X, d)$ and $(X, p)$ be a metric space and partial metric space, respectively.

Lemma 1. Mappings $\rho_{i}: X \times X \longrightarrow \mathbb{R}^{+}(i \in\{1,2,3\})$ defined by

$$
\begin{aligned}
& \rho_{1}(x, y)=d(x, y)+p(x, y) \\
& \rho_{2}(x, y)=d(x, y)+\max \{\omega(x), \omega(y)\} \\
& \rho_{3}(x, y)=d(x, y)+a
\end{aligned}
$$

define partial metrics on $X$, where $\omega: X \longrightarrow \mathbb{R}^{+}$is an arbitrary function and $a \geq 0$.

In addition in above Lemma the partial metric $\rho_{3}$ is a special case of $\rho_{2}$, and $\rho_{2}$ is a special case of $\rho_{1}$.

Each partial metric $p$ on $X$ generates a $T_{0}$ topology $\tau_{p}$ on $X$ which has as a base the family of open $p$ - balls

$$
\left\{B_{p}(x, \varepsilon): x \in X, \varepsilon>0\right\},
$$

where $B_{p}(x, \varepsilon)=\{y \in X: p(x, y)<p(x, x)+\varepsilon\}$ for all $x \in X$ and $\varepsilon>0$.

Definition 1. A subset $A$ of a partial metric space $(X, p)$ is called open if for every $x \in A$ there exists $r>0$ such that $B_{p}(x, r) \subset A$.

Let $\tau_{p}$ be the set of all open subsets $X$, then $\tau_{p}$ is a topology on $X$ (induced by the partial metric $p$ ).

Definition 2. A sequence $\left\{x_{n}\right\}$ in a partial metric space $(X, p)$ converges to $x$ if and only if $p(x, x)=\lim _{n \rightarrow \infty} p\left(x_{n}, x\right)$. That is, for each $\varepsilon>0$ there exists $n_{0} \in \mathbb{N}$ such that

$$
p\left(x, x_{n}\right)<p(x, x)+\varepsilon \quad \forall n \geq n_{0} .
$$


Definition 3. A sequence $\left\{x_{n}\right\}$ in a partial metric $(X, p)$ is called a Cauchy sequence if there exists $\lim _{n, m \rightarrow \infty} p\left(x_{n}, x_{m}\right)$ which is finite.

A partial metric space $(X, p)$ is said to be complete if every Cauchy sequence $\left\{x_{n}\right\}$ in $X$ converges, with respect to $\tau_{p}$, to a point $x \in X$ such that $p(x, x)=$ $\lim _{n, m \rightarrow \infty} p\left(x_{n}, x_{m}\right)$.

Suppose that $\left\{x_{n}\right\}$ is a sequence in partial metric space $(X, p)$, then we define $L\left(x_{n}\right)=\left\{x \mid x_{n} \longrightarrow x\right\}$.

The following example shows that every convergent sequence $\left\{x_{n}\right\}$ in a partial metric space $X$ may not be Cauchy. In particular, it shows that the limit of a convergent sequence is not unique.

Example 1. Let $X=[0, \infty)$ and $p(x, y)=\max \{x, y\}$. Let

$$
x_{n}= \begin{cases}0, & n=2 k \\ 1, & n=2 k+1 .\end{cases}
$$

Then clearly it is convergent sequence and for every $x \geq 1$ we have $\lim _{n \rightarrow \infty} p\left(x_{n}, x\right)=$ $p(x, x)$, therefore $L\left(x_{n}\right)=[1, \infty)$. But $\lim _{n, m \rightarrow \infty} p\left(x_{n}, x_{m}\right)$ does not exist.

The following Lemma shows that under certain conditions the limit is unique.

Lemma 2. Let $\left\{x_{n}\right\}$ be a convergent sequence in partial metric space $X$ such that $x_{n} \longrightarrow x$ and $x_{n} \longrightarrow y$. If

$$
\lim _{n \rightarrow \infty} p\left(x_{n}, x_{n}\right)=p(x, x)=p(y, y),
$$

then $x=y$.

Proof. As

$$
p(x, y) \leq p\left(x, x_{n}\right)+p\left(x_{n}, y\right)-p\left(x_{n}, x_{n}\right),
$$

therefore

$$
p\left(x_{n}, x_{n}\right) \leq p\left(x, x_{n}\right)+p\left(x_{n}, y\right)-p(x, y) .
$$

By given assumptions, we have $\lim _{n \rightarrow \infty} p\left(x_{n}, x\right)=p(x, x), \lim _{n \rightarrow \infty} p\left(x_{n}, y\right)=p(y, y)$, and $\lim _{n \rightarrow \infty} p\left(x_{n}, x_{n}\right)=p(x, x)$. Therefore

$$
p(x, x) \leq p(x, x)+p(y, y)-p(x, y)
$$

which shows that $p(y, y) \leq p(x, y) \leq p(y, y)$. Also,the inequality

$$
p(x, x) \leq p(x, y) \leq p(x, x)
$$

follow directly from the first part of the proof by replacing $\mathrm{x}$ and $\mathrm{y}$, and using property (p3). Thus $p(x, x)=p(x, y)=p(y, y)$, therefore $x=y$. 
Lemma 3. Let $\left\{x_{n}\right\}$ and $\left\{y_{n}\right\}$ be two sequences in partial metric space $X$ such that

$$
\lim _{n \rightarrow \infty} p\left(x_{n}, x\right)=\lim _{n \rightarrow \infty} p\left(x_{n}, x_{n}\right)=p(x, x)
$$

and

$$
\lim _{n \rightarrow \infty} p\left(y_{n}, y\right)=\lim _{n \rightarrow \infty} p\left(y_{n}, y_{n}\right)=p(y, y)
$$

then $\lim _{n \rightarrow \infty} p\left(x_{n}, y_{n}\right)=p(x, y)$. In particular, $\lim _{n \rightarrow \infty} p\left(x_{n}, z\right)=p(x, z)$ for every $z \in$ $X$.

Proof. As $\left\{x_{n}\right\}$ and $\left\{y_{n}\right\}$ converge to a $x \in X$ and $y \in X$ respectively, therefore for each $\epsilon>0$ there exist $n_{0} \in \mathbb{N}$ such that

$$
p\left(x, x_{n}\right)<p(x, x)+\frac{\epsilon}{2}, p\left(y, y_{n}\right)<p(y, y)+\frac{\epsilon}{2}, p\left(x, x_{n}\right)<p\left(x_{n}, x_{n}\right)+\frac{\epsilon}{2}
$$

and

for $n \geq n_{0}$. Now

$$
p\left(y, y_{n}\right)<p\left(y_{n}, y_{n}\right)+\frac{\epsilon}{2}
$$

$$
\begin{aligned}
p\left(x_{n}, y_{n}\right) & \leq p\left(x_{n}, x\right)+p\left(x, y_{n}\right)-p(x, x) \\
& \leq p\left(x_{n}, x\right)+p(x, y)+p\left(y, y_{n}\right)-p(y, y)-p(x, x) \\
& <p(x, y)+\frac{\epsilon}{2}+\frac{\epsilon}{2}=p(x, y)+\epsilon,
\end{aligned}
$$

and so we have

$$
p\left(x_{n}, y_{n}\right)-p(x, y)<\epsilon .
$$

Also,

$$
\begin{aligned}
p(x, y) & \leq p\left(x, x_{n}\right)+p\left(x_{n}, y\right)-p\left(x_{n}, x_{n}\right) \\
& \leq p\left(x, x_{n}\right)+p\left(x_{n}, y_{n}\right)+p\left(y_{n}, y\right)-p\left(y_{n}, y_{n}\right)-p\left(x_{n}, x_{n}\right) \\
& <\frac{\epsilon}{2}+\frac{\epsilon}{2}+p\left(x_{n}, y_{n}\right)=p\left(x_{n}, y_{n}\right)+\epsilon
\end{aligned}
$$

implies that

$$
p(x, y)-p\left(x_{n}, y_{n}\right)<\epsilon .
$$

Hence for all $n \geq n_{0}$, we have $\left|p\left(x_{n}, y_{n}\right)-p(x, y)\right|<\epsilon$. Hence the result follows.

Lemma 4. If $p$ is a partial metric on $X$, then mappings $p^{s}, p^{m}: X \times X \rightarrow \mathbb{R}^{+}$ given by

$$
p^{s}(x, y)=2 p(x, y)-p(x, x)-p(y, y)
$$

and

$$
p^{m}(x, y)=\max \{p(x, y)-p(x, x), p(x, y)-p(y, y)\}
$$

define equivalent metrics on $X$. 
Proof. It is easy to see that $p^{s}$ and $p^{m}$ are metrics on $X$. Obviously,

$$
p^{m}(x, y) \leq p^{s}(x, y)
$$

for every $x, y \in X$. As for every nonneative real numbers $a$ and $b$, we have $a+b \leq$ $2 \max \{a, b\}$, therefore

$$
\begin{aligned}
p^{s}(x, y) & =2 p(x, y)-p(x, x)-p(y, y) \\
& \leq 2 \max \{p(x, y)-p(x, x), p(x, y)-p(y, y)\}=2 p^{m}(x, y) .
\end{aligned}
$$

Hence

$$
\frac{1}{2} p^{s}(x, y) \leq p^{m}(x, y) \leq p^{s}(x, y) .
$$

So $p^{s}$ and $p^{m}$ are equivalent.

Lemma 5 ([8],[9]). Let $(X, p)$ be a partial metric space.

(a) $\left\{x_{n}\right\}$ is a Cauchy sequence in $(X, p)$ if and only if it is a Cauchy sequence in the metric space $\left(X, p^{s}\right)$.

(b) A partial metric space $(X, p)$ is complete if and only if the metric space $\left(X, p^{s}\right)$ is complete. Furthermore, $\lim _{n \rightarrow \infty} p^{s}\left(x_{n}, x\right)=0$ if and only if

$$
p(x, x)=\lim _{n \rightarrow \infty} p\left(x_{n}, x\right)=\lim _{n, m \rightarrow \infty} p\left(x_{n}, x_{m}\right) .
$$

Lemma 6. If $\left\{x_{n}\right\}$ is a convergent sequence in $\left(X, p^{s}\right)$, then it is a convergent sequence in the partial metric space $(X, p)$.

Proof. As, $\lim _{n \rightarrow \infty} p^{s}\left(x_{n}, x\right)=0$, and $p\left(x_{n}, x_{n}\right) \leq p\left(x_{n}, x\right)$.Therefor

$$
0 \leq p\left(x_{n}, x\right)-p(x, x) \leq p^{s}\left(x_{n}, x\right)
$$

implies that

$$
0 \leq \limsup _{n \rightarrow \infty} p\left(x_{n}, x\right)-p(x, x) \leq \lim _{n \rightarrow \infty} p^{s}\left(x_{n}, x\right)
$$

and consequently, $\lim _{n \rightarrow \infty} p\left(x_{n}, x\right)=p(x, x)$.

\section{MAin Result}

We begin this section giving the concept of weakly increasing mappings (see [5]).

Definition 4. Let $(X, \preceq)$ be a partially ordered set. Two mappings $S, T: X \longrightarrow X$ are said to be weakly increasing if $S x \preceq T S x$ and $T x \preceq S T x$ for all $x \in X$.

Note that, two weakly increasing mappings need not be nondecreasing. There exist some examples to illustrate this fact in [3].

Definition 5. Let $(X, \preceq)$ be a partially ordered set. If there exists a partial metric $\mathrm{p}$ in $\mathrm{X}$ such that $x \preceq y$ implies that $p(x, x) \leq p(y, y)$ for $x, y \in X$ then we said that partial metric $\mathrm{p}$ have $\mathrm{p}$-property. 
Example 2. Let $X=\mathbb{R}^{+}$and $\leq$be a partially ordered on $X$. Define $p(x, y)=$ $\max \{x, y\}$. It is easy to see that partial metric $\mathrm{p}$ have $\mathrm{p}$-property.

Example 3. Let $X=\mathbb{R}^{+}$and $\leq$be a partially ordered on $X$. Define $p(x, y)=$ $\max \left\{\left(\frac{1}{2}\right)^{x},\left(\frac{1}{2}\right)^{y}\right\}$. It is easy to see that partial metric $\mathrm{p}$ have not p-property.

Lemma 7. Let $(X, p)$ be a partial metric space and $\varphi: X \longrightarrow \mathbb{R}$. Define the relation $\preceq$ on $X$ as follows:

$$
x \preceq y \Longleftrightarrow x=y \text { or } \quad p(x, y)-p(x, x) \leq \varphi(x)-\varphi(y) .
$$

Then $\preceq$ is a (partial) order on $X$ induced by $\varphi$.

Proof. i) Since $x=x$ hence $x \preceq x$.

ii) Let $x \preceq y$ then $p(x, y)-p(x, x) \leq \varphi(x)-\varphi(y)$. Also, if $y \preceq x$ then $p(x, y)-$ $p(y, y) \leq \varphi(y)-\varphi(x)$. Therefore,

$$
p^{s}(x, y)=2 p(x, y)-p(x, x)-p(y, y) \leq 0,
$$

thus $x=y$.

iii) Let $x \preceq y$ then $p(x, y)-p(x, x) \leq \varphi(x)-\varphi(y)$. Also, if $y \preceq z$ then $p(y, z)-$ $p(y, y) \leq \varphi(y)-\varphi(z)$. Therefore,

$$
p(x, z) \leq p(x, y)+p(y, z)-p(y, y) \leq \varphi(x)-\varphi(z)-p(x, x),
$$

thus $x \preceq z$.

Example 4. Let $X=\{a, b, c\}$. Define $p(a, a)=1, p(b, b)=2, p(c, c)=4, p(a, b)=$ $p(b, a)=2, p(a, c)=p(c, a)=4, p(b, c)=p(c, b)=4$. Define $\varphi(a)=5, \varphi(b)=$ $3, \varphi(c)=1$. It is easy to see that $a \preceq b \preceq c$. That is $\preceq$ is a (partial) order on X induced by $\varphi$.

Theorem 1. Let $(X, p)$ be a complete partial metric space such that partial metric $p$ in $X$ have p-property and $\varphi: X \longrightarrow \mathbb{R}$ be a function which is bounded from below and $\preceq$ the order introduced by $\varphi$. Let $T: X \longrightarrow 2^{X}$ be a multivalued mapping, $[x, \infty)=\{y \in X: x \preceq y\}$ and $M=\{x \in X \mid T(x) \cap[x, \infty) \neq \varnothing\}$. Suppose that

(i) $T$ is upper semicontinuous, that is, $x_{n} \in X$ and $y_{n} \in T\left(x_{n}\right)$ with $x_{n} \longrightarrow x_{0}$ and $y_{n} \longrightarrow y_{0}$, implies $y_{0} \in T\left(x_{0}\right)$,

(ii) $M \neq \varnothing$,

(iii) for each $x \in M, T(x) \cap M \cap[x, \infty) \neq \varnothing$.

Then $T$ has a fixed point $x^{*}$ and there exists a sequence $\left\{x_{n}\right\}$ with

$$
x_{n-1} \preceq x_{n} \in T\left(x_{n}\right), \quad n=1,2,3, \cdots
$$

such that $x_{n} \longrightarrow x^{*}$. Moreover if $\varphi$ is lower semicontinuous, then $x_{n} \preceq x^{*}$ for all $n$.

Proof. By the condition (ii), take $x_{0} \in M$. From (iii), there exist $x_{1} \in T\left(x_{0}\right) \cap M$ and $x_{0} \preceq x_{1}$. Again from (iii), there exist $x_{2} \in T\left(x_{1}\right) \cap M$. Thus $x_{1} \preceq x_{2}$. Continuing this procedure we get a sequence $\left\{x_{n}\right\}$ satisfying

$$
x_{n-1} \preceq x_{n} \in T\left(x_{n-1}\right), \quad n=1,2,3, \cdots .
$$


So by the definition of $\preceq$, we have

$$
0 \leq p\left(x_{n-1}, x_{n}\right)-p\left(x_{n-1}, x_{n-1}\right) \leq \varphi\left(x_{n-1}\right)-\varphi\left(x_{n}\right),
$$

that is $\cdots \varphi\left(x_{2}\right) \leq \varphi\left(x_{1}\right) \leq \varphi\left(x_{0}\right)$, that is, the sequence $\left\{\varphi\left(x_{n}\right)\right\}$ is a nonincreasing sequence in $\mathbb{R}$. Since $\varphi$ is bounded from below, $\left\{\varphi\left(x_{n}\right)\right\}$ is convergent and hence it is Cauchy, that is, for all $\varepsilon>0$, there exists $n_{0} \in \mathbb{N}$ such that for all $m>n>n_{0}$ we have $\left|\varphi\left(x_{m}\right)-\varphi\left(x_{n}\right)\right|<\varepsilon$. Since $x_{n} \preceq x_{m}$, we have $x_{n}=x_{m}$ or $p\left(x_{n}, x_{m}\right)-p\left(x_{n}, x_{n}\right) \leq$ $\varphi\left(x_{n}\right)-\varphi\left(x_{m}\right)$. Therefore,

$$
\begin{aligned}
p\left(x_{n}, x_{m}\right)-p\left(x_{n}, x_{n}\right) & \leq \varphi\left(x_{n}\right)-\varphi\left(x_{m}\right) \\
& =\left|\varphi\left(x_{n}\right)-\varphi\left(x_{m}\right)\right| \\
& <\varepsilon .
\end{aligned}
$$

On the other hand, since partial metric $\mathrm{p}$ has p-property we have

$$
p\left(x_{n}, x_{m}\right)-p\left(x_{m}, x_{m}\right) \leq p\left(x_{n}, x_{m}\right)-p\left(x_{n}, x_{n}\right)<\varepsilon .
$$

Therefore

$$
\begin{aligned}
p^{S}\left(x_{n}, x_{m}\right) & =2 p\left(x_{n}, x_{m}\right)-p\left(x_{n}, x_{n}\right)-p\left(x_{m}, x_{m}\right) \\
& <2 \varepsilon .
\end{aligned}
$$

That is $\left\{x_{n}\right\}$ is a Cauchy sequence in the metric space $\left(X, p^{S}\right)$. Since $(X, p)$ is complete then from Lemma 5, the sequence $\left\{x_{n}\right\}$ converges in the metric space $\left(X, p^{s}\right)$, say $\lim _{n \rightarrow \infty} p^{s}\left(x_{n}, x^{*}\right)=0$. Again from Lemma 5, we have

$$
p\left(x^{*}, x^{*}\right)=\lim _{n \rightarrow \infty} p\left(x_{n}, x^{*}\right)=\lim _{n, m \rightarrow \infty} p\left(x_{n}, x_{m}\right) .
$$

Since $\mathrm{T}$ is upper semicontinuous, $x^{*} \in T\left(x^{*}\right)$.

Moreover, when $\varphi$ is lower semicontinuous, by Lemma 3 and above equality for each $n$ we have:

$$
\begin{aligned}
p\left(x_{n}, x^{*}\right)-p\left(x_{n}, x_{n}\right) & =\lim _{m \rightarrow \infty} p\left(x_{n}, x_{m}\right)-p\left(x_{n}, x_{n}\right) \\
& \leq \lim _{m \rightarrow \infty} \sup \left(\varphi\left(x_{n}\right)-\varphi\left(x_{m}\right)\right) \\
& =\varphi\left(x_{n}\right)-\lim _{m \rightarrow \infty} \inf \varphi\left(x_{m}\right) \\
& \leq \varphi\left(x_{n}\right)-\varphi\left(x^{*}\right) .
\end{aligned}
$$

So $x_{n} \preceq x^{*}$, for all $\mathrm{n}$.

Similarly, we can prove the following.

Theorem 2. Let $(X, p)$ be a complete partial metric space such that partial metric $p$ in $X$ have p-property and $\varphi: X \longrightarrow \mathbb{R}$ be a function which is bounded from below and $\preceq$ the order introduced by $\varphi$. Let $T: X \longrightarrow 2^{X}$ be a multivalued mapping, $(-\infty, x]=\{y \in X: y \preceq x\}$ and $M=\{x \in X \mid T(x) \cap(-\infty, x] \neq \varnothing\}$. Suppose that 
(i) $T$ is upper semicontinuous, that is, $x_{n} \in X$ and $y_{n} \in T\left(x_{n}\right)$ with $x_{n} \longrightarrow x_{0}$ and $y_{n} \longrightarrow y_{0}$, implies $y_{0} \in T\left(x_{0}\right)$,

(ii) $M \neq \varnothing$,

(iii) for each $x \in M, T(x) \cap M \cap(-\infty, x] \neq \varnothing$.

Then $T$ has a fixed point $x^{*}$ and there exists a sequence $\left\{x_{n}\right\}$ with

$$
x_{n-1} \succeq x_{n} \in T\left(x_{n-1}\right), \quad n=1,2,3, \cdots
$$

such that $x_{n} \longrightarrow x^{*}$. Moreover if $\varphi$ is upper semicontinuous, then $x^{*} \preceq x_{n}$ for all $n$.

Theorem 3. Let $(X, p)$ be a complete partial metric space such that partial metric $p$ in $X$ have p-property and $\varphi: X \longrightarrow \mathbb{R}$ be a function which is bounded below and $\preceq$ the order introduced by $\varphi$. Let $S, T: X \longrightarrow X$ are two weakly increasing mappings, then

(i) there exists a sequence $\left\{x_{n}\right\}$ with

$$
x_{n} \preceq x_{n+1}, \quad n=1,2,3, \cdots \text { and } \lim _{n \rightarrow \infty} S x_{2 n}=x^{*} \quad \lim _{n \rightarrow \infty} T x_{2 n+1}=x^{*} \text {, }
$$

(ii) if $S, T$ are continuous in $\left(X, p^{S}\right)$ then $x^{*}$ is common fixed point of $S, T$.

Proof. Let $x_{0}$ be an arbitrary point of X. Set $S x_{0}=x_{1}$ and $T x_{1}=x_{2}$. We can define a sequence in $\mathrm{X}$ as follows:

$$
S x_{2 n}=x_{2 n+1} \text { and } T x_{2 n+1}=x_{2 n+2} \text { for } n \in\{0,1, \cdots\} \text {. }
$$

Note that, since $\mathrm{S}$ and $\mathrm{T}$ are weakly increasing, we have

$$
x_{1}=S x_{0} \preceq T S x_{0}=T x_{1}=x_{2} \preceq S T x_{1}=S x_{2}=x_{3}
$$

and continuing this process we have

$$
x_{1} \preceq x_{2} \preceq \cdots \preceq x_{n} \preceq x_{n+1} \preceq \cdots .
$$

So by the definition of $\preceq$, we have

$$
0 \leq p\left(x_{n}, x_{n+1}\right)-p\left(x_{n}, x_{n}\right) \leq \varphi\left(x_{n}\right)-\varphi\left(x_{n+1}\right),
$$

that is $\cdots \varphi\left(x_{n+1}\right) \leq \varphi\left(x_{n}\right)$, that is, the sequence $\left\{\varphi\left(x_{n}\right)\right\}$ is a nonincreasing sequence in $\mathbb{R}$. Since $\varphi$ is bounded from below, $\left\{\varphi\left(x_{n}\right)\right\}$ is convergent and hence it is Cauchy, that is, for all $\varepsilon>0$, there exists $n_{0} \in \mathbb{N}$ such that for all $m>n>n_{0}$ we have $\left|\varphi\left(x_{m}\right)-\varphi\left(x_{n}\right)\right|<\varepsilon$. Since $x_{n} \preceq x_{m}$, we have $x_{n}=x_{m}$ or $p\left(x_{n}, x_{m}\right)-p\left(x_{n}, x_{n}\right) \leq$ $\varphi\left(x_{n}\right)-\varphi\left(x_{m}\right)$. Therefore,

$$
\begin{aligned}
p\left(x_{n}, x_{m}\right)-p\left(x_{n}, x_{n}\right) & \leq \varphi\left(x_{n}\right)-\varphi\left(x_{m}\right) \\
& =\left|\varphi\left(x_{n}\right)-\varphi\left(x_{m}\right)\right| \\
& <\varepsilon .
\end{aligned}
$$

On the other hand, since partial metric p has p-property we have

$$
p\left(x_{n}, x_{m}\right)-p\left(x_{m}, x_{m}\right) \leq p\left(x_{n}, x_{m}\right)-p\left(x_{n}, x_{n}\right)<\varepsilon .
$$


Therefore

$$
\begin{aligned}
p^{s}\left(x_{n}, x_{m}\right) & =2 p\left(x_{n}, x_{m}\right)-p\left(x_{n}, x_{n}\right)-p\left(x_{m}, x_{m}\right) \\
& <2 \varepsilon .
\end{aligned}
$$

That is $\left\{x_{n}\right\}$ is a Cauchy sequence in the metric space $\left(X, p^{s}\right)$. Since $(X, p)$ is complete then from Lemma 5 , the sequence $\left\{x_{n}\right\}$ converges in the metric space $\left(X, p^{s}\right)$, say $\lim _{n \rightarrow \infty} p^{s}\left(x_{n}, x^{*}\right)=0$. Again from Lemma 5, we have

$$
p\left(x^{*}, x^{*}\right)=\lim _{n \rightarrow \infty} p\left(x_{n}, x^{*}\right)=\lim _{n, m \rightarrow \infty} p\left(x_{n}, x_{m}\right) .
$$

(ii)

$$
S x^{*}=\lim _{n \rightarrow \infty} S x_{2 n}=\lim _{n \rightarrow \infty} x_{2 n+1}=x^{*},
$$

and

$$
T x^{*}=\lim _{n \rightarrow \infty} T x_{2 n+1}=\lim _{n \rightarrow \infty} x_{2 n+2}=x^{*} .
$$

Example 5. Now, consider the $X=[0,1]$. Define $\varphi: X \longrightarrow \mathbb{R}$ by $\varphi(x)=1-x$ and define $S, T: X \longrightarrow X$ by $S x=\frac{x+1}{2}$ and $T x=\frac{2 x+1}{3}$. If $p(x, y)=\max \{x, y\}$ then it is easy to see that $\leq$ is a partial order relation on $X$ induced by $\varphi, T x \leq S T x$ and $S x \leq T S x$, that is $S, T$ are two weakly increasing mappings.

Hence all of the conditions of Theorem 3 are hold. That is there exists $x^{*}=1 \in X$ such that $T x^{*}=S x^{*}=x^{*}=1$.

\section{REFERENCES}

[1] I. Altun and M. Imdad, "Some fixed point theorems on ordered uniform space," Filomat, vol. 23, no. 3, pp. 15-22, 2009.

[2] I. Altun and H. Simsek, "Some fixed point theorems on dualistic partial metric spaces," J. Adv. Math. Stud., vol. 1, no. 1-2, pp. 1-8, 2008.

[3] I. Altun and H. Simsek, "Some fixed point theorems on ordered metric spaces and application," Fixed Point Theory Appl., vol. 2010, p. 17, 2010.

[4] I. Altun, F. Sola, and H. Simsek, "Generalized contractions on partial metric spaces," Topology Appl., vol. 157, no. 18, pp. 2778-2785, 2010.

[5] I. Beg and A. R. Butt, "Fixed point for set-valued mappings satisfying an implicit relation in partially ordered metric spaces," Nonlinear Anal., Theory Methods Appl., vol. 71, no. 9, pp. A, 3699-3704, 2009.

[6] R. Heckmann, "Approximation of metric spaces by partial metric spaces," Appl. Categ. Struct., vol. 7, no. 1-2, pp. 71-83, 1999.

[7] M. Hötzel Escardó, "PCF extended with real numbers," Theor. Comput. Sci., vol. 162, no. 1, pp. 79-115, 1996.

[8] S. G. Matthews, "Partial metric topology," in Papers on general topology and applications, ser. Papers from the 8th summer conference at Queens College, New York, NY, USA, June 18-20, 1992, S. Andima, Ed. New York: The New York Academy of Sciences, 1994, vol. 728, pp. 183-197. 
[9] S. Oltra and O. Valero, "Banach's fixed point theorem for partial metric spaces," Rend. Ist. Mat. Univ. Trieste, vol. 36, no. 1-2, pp. 17-26, 2004.

[10] S. Romaguera, "A Kirk type characterization of completeness for partial metric spaces," Fixed Point Theory Appl., vol. 2010, p. 6, 2010.

[11] O. Valero, "On Banach fixed point theorems for partial metric spaces," Appl. Gen. Topol., vol. 6, no. 2, pp. 229-240, 2005.

[12] X. Zhang, "Fixed point theorems of multivalued monotone mappings in ordered metric spaces," Appl. Math. Lett., vol. 23, no. 3, pp. 235-240, 2010.

Authors' addresses

Shaban Sedghi

Department of Mathematics, Qaemshahr Branch, Islamic Azad University, Qaemshahr, Iran

E-mail address: sedghi_gh@yahoo.com, sedghi.gh@qaemshahriau.ac.ir

\section{Nabiollah Shobkolaei}

Department of Mathematics,, Islamic Azad University, Science and Research Branch, 1477893855

Tehran, Iran

E-mail address: nabi_shobe@yahoo.com

\section{Duran Turkoglu}

Department of Mathematics, Faculty of Science and Arts, University of Gazi, 06500-Teknikokullar, Ankara, Turkey, Department of Mathematics, Faculty of Science and Arts, University of Amasya, Amasya, Turkey

E-mail address: dturkoglu@gazi.edu.tr 